\title{
Proton radii and two-photon exchange
}

\author{
Peter G. Blunden \\ Dept. of Physics and Astronomy, University of Manitoba, Winnipeg, MB, Canada R3T 2N2 \\ Ingo Sick \\ Dept. für Physik und Astronomie, Universität Basel, CH4056 Basel, Switzerland
}

(Dated: May 20, 2018)

\begin{abstract}
We investigate the effect of two-photon exchange processes upon the rms- and Zemach radii extracted from electron-proton scattering. We find that the changes are small and do not help to explain the discrepancy between experimental and calculated HFS in the hydrogen atom.
\end{abstract}

PACS numbers: 25.30.Bf, 21.20.Ft, 14.20.Dh

\section{INTRODUCTION}

The recent progress in measurements of transition energies in the Hydrogen atom has been remarkable. The $2 \mathrm{p}-1 \mathrm{~s}$ transition energy and the 1s hyperfine structure interval (HFS) are now known to 14 and 12 significant digits, respectively 1, 2]. The interpretation of these energies in terms of e.g. tests of QED now depends entirely on the accuracy with which the proton finite-size corrections are known.

These finite-size corrections can be determined from elastic electron-proton scattering at low momentum transfer $q$. The proton moment relevant for the $2 \mathrm{p}-1 \mathrm{~s}$ energy (and the Lamb shift) is the charge rms-radius $r_{r m s}$ which has been extracted from the world data on e-p scattering 3. to $0.895 \pm 0.018 \mathrm{fm}$. With this radius as input, calculated and experimental $2 \mathrm{p}-1$ s transition energies agree within the error bars. The proton moment relevant for the HFS is the Zemach moment, derived from a convolution of charge- and magnetization densities. This radius has also been extracted from the world e-p data in Ref. [4] which found $\langle r\rangle_{(2)}=1.086 \pm 0.012 \mathrm{fm}$. The corresponding calculated HFS disagrees with experiment by $3.6(5) \mathrm{ppm}$ [4] (see also [5, 6, 7]), where the uncertainty is dominated by the uncertainty in $\langle r\rangle_{(2)}$.

This disagreement is partly explained by nuclear polarization effects in the hydrogen atom, processes involving virtual excitation of the proton to intermediary continuum states. Faustov et al. 8] have calculated this correction and find a contribution of $1.6 \mathrm{ppm}$ in the right direction using the experimental $g_{1}(q)$ and $g_{2}(q)$ spin structure functions of the proton. The uncertainty of this correction is hard to estimate as the nuclear polarization correction depends on $g_{1}, g_{2}$ at very low $q$, where these functions are poorly known.

Before assigning the remaining discrepancy to this correction (or to not yet calculated higher-order QED terms affecting the HFS) one should note, however, that the moments extracted from electron scattering are based on an interpretation of the data in one-photon exchange (plus exchange of additional soft photons responsible for the Coulomb distortion of the electron waves $[9]$ ). Electron scattering is also subject to exchange of two hard photons, which e.g. have a considerable effect upon the proton charge form factor $G_{E}$ as determined from longitudinal/transverse separations at very large momentum transfers [10].

In this Rapid Communication, we investigate the role of the two-photon exchange in the determination of the proton moments, in order to find out whether these corrections could be responsible for the discrepancy with the HFS values.

\section{CALCULATION OF TWO-PHOTON EXCHANGE}

Details of the two-photon exchange correction to elastic electron-proton scattering are described elsewhere [10, 11]. We consider the contribution to the two-photon exchange box and crossed-box amplitudes with an intermediate nucleon. At the low momentum transfers of interest here (up to $q=4 \mathrm{fm}^{-1}$ ) the contribution of an intermediate $\Delta$ (or higher resonances) to the two-photon exchange amplitude is negligible [12].

Hadronic form factors consistent with the experimentally measured charge and magnetic form factors $G_{E}\left(q^{2}\right)$ and $G_{M}\left(q^{2}\right)$ are introduced at the photon-nucleon vertices. The two-photon exchange correction is relatively insensitive to the particular choice of hadronic form factors 11. For very low $q$, the two-photon exchange correction behaves the same as that found for scattering from a point particle (e.g. electron-muon scattering), and is therefore completely independent of the hadronic form factors in this limit. This is a useful check on our calculation.

In order to give an impression of the calculated results, we show in Fig. 1] the two-photon correction for a typical electron energy. The two-photon exchange contribution is compared to the contribution involving only the piece from a second soft photon (Coulomb distortion of the electron wave), calculated according to Ref. 9] in second Born approximation. The same contribution, but for a point-nucleus, is given for comparison. Fig. 11 shows that at forward angles the two-photon contribution is entirely dominated by Coulomb distortion, while at backward angles the exchange of two hard photons 


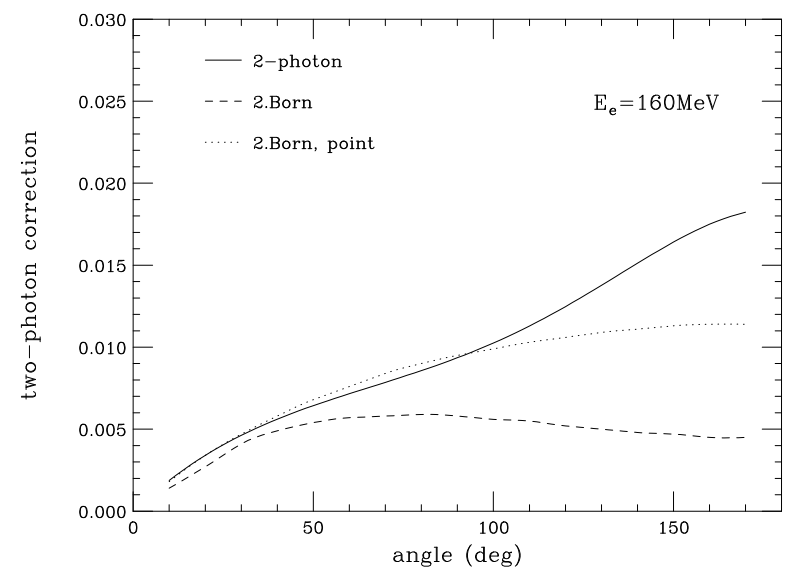

FIG. 1: Relative contribution of two-photon exchange to elastic e-p scattering at $\mathrm{E}=160 \mathrm{MeV}$. The results in second Bornapproximation account for the Coulomb distortion (exchange of second soft photon) only.

contributes appreciably.

\section{ANALYSIS OF WORLD E-P DATA}

It has been previously shown in Ref. [3] that for an optimal determination of the proton moments a parameterization in terms of a Continued Fraction (CF) expansion should be used. For this parameterization, the contribution of model-dependence has been investigated in detail. As in Ref. 3] we use the cross sections up to a maximum momentum transfer of $4 \mathrm{fm}^{-1}$.

We use the world cross sections on e-p scattering (for references see [3]). These data have been corrected for the contribution of two-photon effects (omitting the second Born Coulomb corrections, which are already included in the two-photon contribution), and then fitted with 5parameter $\mathrm{CF}$ expressions for both the charge and the magnetic form factors $G_{E}\left(q^{2}\right)$ and $G_{M}\left(q^{2}\right)$. The two- photon corrected longitudinal/transverse separation is thus done implicitly during the fit. The charge-rms radius is obtained from the slope at $q^{2}=0$ of $G_{E}\left(q^{2}\right)$, the Zemach moment is obtained via

$$
\langle r\rangle_{(2)}=-\frac{4}{\pi} \int_{0}^{\infty} \frac{d q}{q^{2}}\left(G_{E}\left(q^{2}\right) G_{M}\left(q^{2}\right)-1\right) .
$$

The statistical errors have been determined using the error matrix, the systematic errors have been obtained by changing the data sets individually by their systematic errors, refitting the data and adding quadratically all the resulting changes of the moments. The contribution of the model dependence is accounted for as well.

\section{RESULTS AND CONCLUSION}

The change of $\langle r\rangle_{(2)}$ found when removing the contribution of two-photon exchange (beyond Coulomb distortion) amounts to $+0.0052 \mathrm{fm}$. This change is small ( $\sim 40 \%$ of the error bar) and goes in the wrong direction in terms of helping to explain the HFS discrepancy. The Zemach moment with two-photon effects corrected for amounts to $=1.091 \pm 0.012 \mathrm{fm}$. The change in the charge-rms radius, $+0.0015 \mathrm{fm}$, is also small. The radius after two-photon correction amounts to $0.897 \pm 0.018 \mathrm{fm}$.

From these results we conclude that the discrepancy between calculated and experimental HFS in the hydrogen atom cannot be attributed to two-photon exchange contributions to (e,e) that could have falsified the Zemach moment from e-p scattering. The origin of this discrepancy presumably has to be sought in the uncertain nuclear polarization correction to HFS, or potentially not yet calculated higher-order contributions to the HFS.

\section{Acknowledgments}

This work has been supported by NSERC (Canada) and by the Schweizerische Nationalfonds.
[1] M. Niering et al. Phys. Rev. Lett., 84:5496, 2000.

[2] M.I. Eides, H. Grotch, and V.A. Shelyuto. Phys. Rep., 342:63, 2001.

[3] I. Sick. Phys. Lett. B, 576:62, 2003.

[4] J.L. Friar and I. Sick. Phys. Lett. B, 579:285, 2004.

[5] S.J. Brodsky, C.E. Carlson, J.R. Hiller, and D.S. Hwang. Phys. Rev. Lett., 94:22001, 2005.

[6] S.J. Brodsky, C.E. Carlson, J.R. Hiller, and D.S. Hwang. Phys. Rev. Lett., 94:169902, 2005.

[7] J.L. Friar and I. Sick. Phys. Rev. Lett., 95:49101, 2005.
[8] R.N. Faustov and A.P. Martynenko. Eur. Phys. C, 24:281, 2002.

[9] I. Sick and D. Trautmann. Nucl. Phys. A, 637:559, 1998.

[10] P.G. Blunden, W. Melnitchouk, and J.A. Tjon. Phys. Rev. Lett., 91:142304, 2003.

[11] P.G. Blunden, W. Melnitchouk, and J.A. Tjon. Nuclth/0506039, 2005.

[12] S. Kondratyuk, P.G. Blunden, W. Melnitchouk, and J.A. Tjon. Nucl-th/0506026, 2005. 